\title{
Visual and Verbal Elements in the Dunkin Donuts Advertisements
}

\author{
Dewa Ayu Kadek Claria ${ }^{1}$, Ni Made Harumdari ${ }^{2}$ \\ Faculty of Letters, Universitas Warmadewa Denpasar-Bali, Indonesia ${ }^{1,2}$ \\ \{claria@warmadewa.ac.id ${ }^{1}$,harumdari@gmail.com² ${ }^{2}$
}

\begin{abstract}
The purpose of this research is to interpret verbal and visual which found in Dunkin Donuts Advertisement. The data were taken from the Dunkin Donuts brochure. The theories applied to support this paper are the theories of advertising. The theories were taken from the book entitled Advertising as Communication [1]. Other books are also used to supoort this writing such as A Semantic [2] about meaning. The research result show that there are found the verbal elements in the form of phrase and sentence of Dunkin Donuts advertisement are in the form of phrase and sentence. Those forms are found in headline, body copy, signature line, and standing details. The types of meaning there are Visual and Verbal Elements are denotative meaning and affective meaning.
\end{abstract}

Keywords: Advertisements; Dunkin Donuts; Visual and Verbal Elements

\section{Introduction}

Discourse analysis or discourse field is the ways to deal with dissect composed, vocal, or language based communication use, or any note worthy semiotic function. Semiotics is the study of meaning-making, the philosophical theory of signs and symbols. This includes the study of signs and sign processes, indication, designation, likeness, analogy, metaphor, symbolism, signification, and communication. Semiotics is concerned with everything that can be taken as a sign. Semiotic expects to take in any arrangement of signs, whatever their substance and cutoff points, pictures, motions, melodic sounds, objects, and the intricate relationship of these, which structure the substance of custom, show or public amusement: these establish, if not dialects, in any event frameworks of connotation. Semiotics is regularly utilized in the examination of writings despite the fact that it is undeniably something beyond a method of printed textual analysis. Here it ought to maybe be noticed that a content can exist in any medium and might be verbal, non-verbal, or both, notwithstanding the logo driven predisposition of this qualification. The term text typically alludes to a message which has been recorded here and there for example composing, sound and video-recording with the goal that it is truly free of its sender or recipient. Saussure (1974) stated that a content is an array of signs, for example, words, pictures, sounds, or potentially gesture developed, and deciphered regarding the shows related with a type and in a specific particular of correspondence. Similarly, [3] in their Discourse Analysis characterize text as - the verbal record of an informative demonstration. Text is delivered and deciphered between two individuals in certain 
unique situation, since people use language to speak with others. Semiotics is a science which contemplates the function of signs as a major aspect of social life, while semiotics is the conventional tenet of signs which is firmly identified with Logic. [4] A sign is something which represents a person or thing in some regard or limit. He proclaimed that each concept is a sign. Moreover, advertisement contains two signs, visual and verbal signs. Visual signs are about pictures and verbal signs are about text, either words or sentences. The use of these two signs in an advertisement is meant to make people understand the product advertised by the producers. [5] Several classifications of signs as, symbols, indexes, and images. A symbol is an indication that is made to resemb1e, recreate, or duplicate its referent. Photos might be notable signs since they can be believed to duplicate their referents in a visual manner. Nothing is a sign except if it is deciphered as a sign. Anything can be a sign as long as somebody deciphers it as implying something - alluding to or representing some different option from itself. It is this important utilization of signs which is at the core semiotics. The advertiser makes a good picture, a good sentence, also artist as the model to their advertisement to persuade the reader to buy their product. [1] The word advertising implies causing to notice something, or telling or advising someone regarding something. However advertisements must also take into account the way in which they can make the characteristics and properties of the items.

Regarding to advertisement, the following section would be pertained some latest related research with this current issue. Firstly, a research who carried out [6] that showed the results that the capacity of mental symbolism adds to upgrade the review of publicizing messages utilizing figures of manner of speaking. Contribution moderatingly affected the connection between logical figures and mentality toward the notice. Secondly, a research conducted [7] which discovered that an insightful "matching" of the visual plan properties utilized in advertisements (predominately visual or verbal) and the customer fragment needs (wanting material to be introduced in a visual or verbal way) has all the earmarks of being basic. The utilization of symbolism impacts purchasers' observation since it builds review, upgrades disposition toward the advanced issue, and influences conduct aims. Visual correspondence stands out since advertisements have gotten so intricate in its endeavors to utilize style to hold onto center and convince customers. Thirdly, a research [8] results that showed that utilizing figures can fundamentally upgrade the viability of print publicizing. Visual figures betterly affect feelings. Verbal figures prompted a more positive mentality toward the promotion and disposition toward the brand.

Based on the explanation above, this present study analyzed verbal and visual sign in Dunkin Donuts advertisements. Dunkin Donuts, presently rebranding its stores as Dunkin', is an American global coffee organization and fast assistance café. Dunkin' is one of the biggest espresso and heated products chains on the world. Its items incorporate doughnuts, bagels, other heated products, and an assortment of hot and frosted drinks. From the explanation above this topic is interesting to be discussed especially verbal and visual elements sign in Dunkin Donuts advertisement. The Dunkin Donuts advertisements are chose to discuss in this research, because Dunkin Donuts is one of most famous of donuts in the world. Although Dunkin Donuts belong to junk food, but Dunkin Donuts is still attract the people with their new food menu. So the Dunkin Donuts introduce their new brand through the advertisement, by showing the picture of their new menu and promotion words. To analyze this topic need to focus not only in one aspect because there are a lot of things to discuss while analyzing this topic especially in visual and verbal of advertisement itself. Verbal and visual sign are important to investigate, because in advertisement contains meaning and message of the advertisement. Each advertisement has meaning and message, the function of those is to attract the people with their product through illustration and some promotion words. Sometime the word on the 
advertisement got confused the reader, so the illustration helps the reader to imagine what the meaning and message of the advertisement. Thus, the aim of this study is to analyze the verbal and visual which found in Dunkin Donuts Advertisement.

\section{Method}

The study is designed by using descriptive qualitative approach through Discourse Analysis method. The methods are applied in determining information source, information assortment and information investigation and concluding the result. The data source in this study was collected from The Dunkin Donuts advertisement. They are in the form of picture containing text and also representing the element of advertisement, such as headline, body copy, standing details, signature line, and illustration. Data collecting is the systematic approach to gather and measure information from a variety of source to get a complete and accurate. The advertisement will read, then the data will collect based on topic, and the data will be classified based on kinds of verbal and visual elements found in each advertisement. The method in collect the data includes observation method by reading the advertisement several times, and note taking, quoting some sentences and identifying the illustrations directly from advertisement which can be considered as the verbal elements and visual elements. In addition, the data in this study would be presented descriptively so that the elaborations about visual and verbal on the Dunkin Donuts advertisement can be clearly explained.

\section{Results and Discussion}

\subsection{The Verbal Elements of Dunkin Donuts Advertisement}

\subsubsection{The Analysis of Verbal Element in Headline}

\section{a) Verbal Element on Headline in the Form of Noun Phrase}

In headline of the Dunkin Donuts Brochure, the headline is found in the form of noun phrase, they are:

The data (1) found in the brochure is "Beverage". The noun phrase of Beverage has denotative meaning because the noun phrase "Beverage" in the brochure means any sort of drink. It's something you may offer a visitor in your home, it's additionally the most loved moniker of organizations that produce both soda and juice - they call themselves refreshment organizations. It has function for giving information about list of what drinks available to the customer about what drinks and at what price.

The data (2) in the brochure is "Snack". The noun phrase of Snack has denotative meaning. The noun phrase of Snack in the brochure means a little assistance of food and for the most part food between meals. snacks arrive in an assortment of structures including bundled snack foods and other handled foods, just as things produced using new ingredients at home. Snack foods are regularly intended to be compact, fast, and fulfilling. Noun phrase of Snack has function in the brochure as the information list of snack or small food about what the snacks available at the company and at what price.

The data (3) in the brochure is "Combos". Noun phrase of Combos has denotative meaning. Noun phrase of Combos also is called as combination meal, it means a sort of meal that 
ordinarily incorporates food things and a drink. They are a typical menu thing at drive-through, and different restaurants additionally furnish them. It has function for persuading the customer about what the package or combo meal offer to the customer and what the combos available at the company and at what price.

\section{b) Verbal Element on Headline in the Form of Sentence}

In headline of Dunkin Donuts advertisement, the headline is found in the form of sentence. The sentence is America Runs on Dunkin. The sentence is categorized as declarative sentence. The sentence America Runs on Dunkin is categorized as declarative sentence because it has structure, America as subject (S), Runs as verb (V), on Dunkin as adverbial (A).

First, the Dunkin Donuts is just justifiable if know the relationship to the organization. The guide of America could be perused as "map" or whatever, yet is significantly additionally fascinating since it is a metonymy. It utilizes "America" to signify "the individuals in America."

The action word "run" pleasantly shows how you can't outwardly show an activity without additionally indicating an item. It's difficult to show "run" without likewise demonstrating the "sprinter." Verbal punctuation (by ethicalness of its emblematic nature) likes to isolate these pieces into [ACTOR]-[ACTION]. In visual language structure this division doesn't function too (being famous, not emblematic), rather turning out to be [ACTOR: state1]-[ACTOR: state2], where "state2" shows the fulfillment of the activity. Indeed, the translation of "run" as an activity here (like "run down the street") is completely off, since they don't imply that Americans "utilize their legs to run on head of Dunkin' Donuts." Rather, they are utilizing a development "run on" (seemingly not two units) that implies generally "to be controlled by." The "individual running" picture at that point turns into a "double rebus" - > first planning the sound example to the picture, at that point the picture's exacting significance to its "connotative" meaning.

\subsubsection{The Analysis of Verbal Element in Body Copy}

Table 1. Verbal Element on Body Copy in the Form of Noun Phrase

\begin{tabular}{|c|c|}
\hline Noun Phrase & Denotative Meaning \\
\hline Cappuccino & A warm cup of blended espresso and frothy, bold deliciousness. \\
\hline Dunkaccino & Made with a mix of coffee, hot chocolate, and milk. \\
\hline Espresso & $\begin{array}{l}\text { It is brewed with freshly ground espresso beans and then blended with } \\
\text { milk and served over ice for a refreshing, frothy and bold iced beverage. }\end{array}$ \\
\hline Hot chocolate & $\begin{array}{l}\text { It means a hot drink made with milk or water and chocolate or } \\
\text { powdered chocolate. }\end{array}$ \\
\hline Hot coffee & $\begin{array}{l}\text { Made by brewing hot water (or much less commonly, cold water) with } \\
\text { ground coffee beans. }\end{array}$ \\
\hline Hot macchiato & $\begin{array}{l}\text { Macchiato means "stained" or "spotted" so the literal translation of caffè } \\
\text { macchiato is "stained coffee", or coffee with a spot of milk. }\end{array}$ \\
\hline Hot tea & $\begin{array}{l}\text { It refers to a hot beverage that is prepared by infusing or } \\
\text { brewing/boiling/decocting the dried leaves of the Camellia Sinensis } \\
\text { plant. }\end{array}$ \\
\hline Frozen coffee coolatta & $\begin{array}{l}\text { It is smooth, creamy and full of real Dunkin flavor - making it the } \\
\text { perfect way to cool off. }\end{array}$ \\
\hline $\begin{array}{l}\text { Minute maid orange } \\
\text { coolatta }\end{array}$ & A variety of fruity flavors that are sure to satisfy. \\
\hline Strawberry coolatta & Fruit grams of sugar mixes with ice. \\
\hline Frozen Dunkacino & Amount of chocolate swirled inside and around the cup. \\
\hline
\end{tabular}




\begin{tabular}{|c|c|}
\hline Noun Phrase & Denotative Meaning \\
\hline Hot chocolate frozen & $\begin{array}{l}\text { An indulgent treat, made with your choice of flavor and topped with } \\
\text { whipped cream. }\end{array}$ \\
\hline $\begin{array}{l}\text { Tropical Mango } \\
\text { Smoothie }\end{array}$ & Made with real fruit and low-fat yogurt mixed with ice and milk. \\
\hline K. Cup pods & It means a beverage brewing system for home and commercial use. \\
\hline Package Coffee & $\begin{array}{l}\text { It means package for coffee which is offering to the customer in Dunkin } \\
\text { Donuts. }\end{array}$ \\
\hline Package Tea & It means Dunkin' Premium Teas can also be enjoyed at home. \\
\hline Donuts single & The customer to try the Donuts single in Dunkin Donuts. \\
\hline Donuts $1 / 2$ Dozen & $\begin{array}{l}\text { It means } \mathbf{1} / 2 \text { dozen or dozen packs Dunkin' Donuts serves many items } \\
\text { such as hot coffee, iced coffee and teas, frozen beverages. }\end{array}$ \\
\hline Donuts Dozen & Its means the dozen may be one of the earliest primitive groupings. \\
\hline Muffins single & It means an individual-sized, baked product. \\
\hline Muffins 4 count & $\begin{array}{l}\text { It means in one package muffin include } 4 \text { single muffins in box with the } \\
\text { price } \$ 3.49 \text {. }\end{array}$ \\
\hline Bagels single & $\begin{array}{l}\text { A bagel is a bread product shaped by hand into the form of a ring from } \\
\text { yeasted wheat dough, roughly hand-sized, that is first boiled for a short } \\
\text { time in water and then baked. }\end{array}$ \\
\hline $\begin{array}{l}\text { Bagels single with } \\
\text { cream }\end{array}$ & $\begin{array}{l}\text { Bagels single with cream is a product which is topped with cream in any } \\
\text { taste. }\end{array}$ \\
\hline Bagels $1 / 2$ dozen & $\begin{array}{l}\text { Bagels 1/2 dozen includes Bagels Plain, Cinnamon Raisin, Sesame, } \\
\text { Everything, Poppy seed, Multigrain, Onion, Wheat. }\end{array}$ \\
\hline
\end{tabular}

\subsubsection{Verbal Element on Body Copy in the Form of Sentence}

In body copy of Dunkin Donuts advertisement, the body copy is found in the form of sentence. The sentence is Dunkin Specialties, your guide to all of the great product of Dunkin' Donuts has to offer. It has structure Dunkin Specialties as subject (S), your guide is as Verb (V), to all of the great product of Dunkin' Donuts has to offer to all of the great product of Dunkin' Donuts has to offer is as complement (C).

Based on the classification of the meaning, the sentence Dunkin Specialties, your guide to all of the great product of Dunkin' Donuts has to offer has an effective meaning, because by using the sentence it can persuade the consumer to try and buy of the great product of Dunkin' Donut has to offer at Dunkin Donuts as their snack menu in every day.

\subsection{The Analysis of Verbal Elements in Standing Details}

\subsubsection{Verbal Elements on Standing Details in the Form of Noun Phrase}

The verbal element found in standing details on the form of noun phrase. Lemon, Cinnamon, Bavarian Kreme, Strawberry Sprinkle, Vanilla Sprinkle, Maple Erosted, Chocolate LongJhon, Vanilla Longjhon, Maple Longjhon, Chocolate Butternut, Peanut, Toasted.

The noun phrase above has denotative meaning because the noun phrase above means many tastes offer for topping of donuts at the Dunkin Donuts. The noun phrase above also has affective meaning, by using the noun phrase Lemon, Cinnamon, Bavarian Kreme, Strawberry Sprinkle, Vanilla Sprinkle, Maple Erosted, Chocolate LongJhon, Vanilla Longjhon, Maple Longjhon, Chocolate Butternut, Peanut, Toasted. It can affect to persuade the consumer for trying the donuts with any kind of topping they want at the Dunkin Donuts. 


\subsubsection{Verbal Elements on Standing Details in the Form of Sentence}

The verbal element found in standing details on the form of sentence. The sentence Visit DDPerks.com or download the Dunkin Mobile App to enroll in DD Perks Today. DDPERKS THAT'S LOYALTY. The sentence has two sentences; they are categorized as imperative sentence because the sentence is preceded by verb. The first sentence is Visit DDPerks.com has structure is a verb (V), DDPerks.com is as object (O). The second sentence is Download the Dunkin Mobile App structure; Download is a verb (V), the Dunkin Mobile App is object (O).

The sentence above has an affective meaning, by visit Dunkin Donuts website it can affect the consumer to see and try some menu in website. The consumer can be ordered by app on app store.

\subsection{The Visual Elements of Dunkin Donuts}

Visual elements of Dunkin Donuts brochure are found in Headline, illustrations, and signature line.

\section{a) The Analysis of Visual Element on Body Copy}

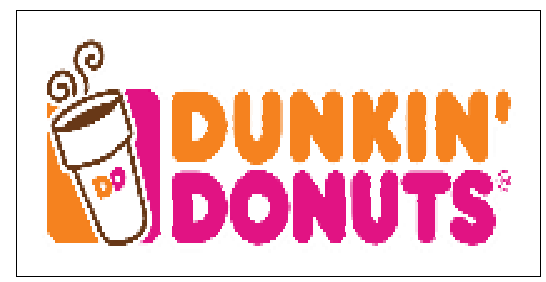

Fig.1. Dunkin Donuts Logo

The logo of Dunkin Donuts is in the global donut company and coffeehouse Dunkin' Donuts adopted its first logo in 1950. The symbol itself depicted a stylized coffee cup with the words "Dunkin' Donuts" given as a circle. In 1976, the orange tone showed up on the token. The changing logo type comprised uniquely of the organization's name.

Notwithstanding the pink-and-orange word mark, the current adaptation, which was embraced in 2006, incorporates a steaming espresso mug laid out in earthy colored with a "DD" monogram. The typeface looks fundamentally the same as the Frankfurter and Debussy textual styles. The letters look full and round to the point of being tantalizing, which appears to be entirely normal for an organization spend significant time in doughnuts. The combination of magenta and orange on the white foundation creates a cheerful, bright, happy mood.

\section{b) The Analysis of Visual Elements on Body Copy}

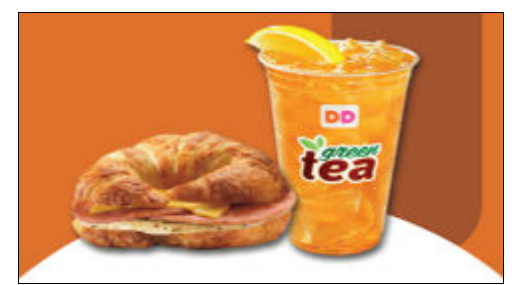

Fig. 2. Picture Iced Lemon Tea and Chicken Bacon Sandwich 
The visual element on the Dunkin Donuts advertisement is also found on the body copy in the form of illustration. There is the picture combos menu, such as Iced Lemon Tea and Chicken Bacon Sandwich. The picture is picture combos menu, there are Iced Lemon Tea and Chicken Bacon Sandwich. The illustration looks delicious, fresh, and cold. The meaning of those illustration is to attract the consumer to try those menus.

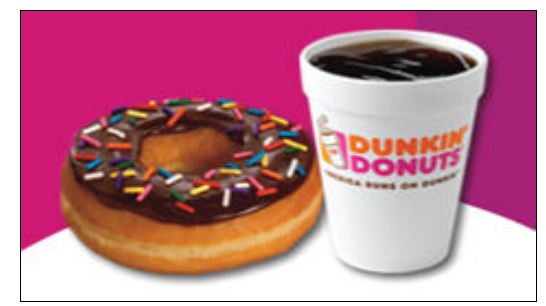

Fig. 3. Picture Single Donut and Iced Tea

The visual element on the Dunkin Donuts advertisement is also found on body copy in the form of illustration. There is the picture snacks package, such as single donut and iced tea. The picture is picture snack package, there are single donut and iced tea. The illustration looks delicious, fresh, and cold. The illustration has an affective meaning of those illustrations is to attract the consumer to try those menus.

\section{Conclusion}

It can be concluded that there are two elements found in Dunkin Donuts advertisement, they are Verbal element and Visual element. The advertisement is about Dunkin Donuts menu which is offering to the consumer. The verbal elements that are found in Dunkin Donuts advertisement which are in the form of pictures, picture of fonts and the logo. Those forms are found in the Headline, Illustration and signature line. The types of meaning that are found in verbal element are denotative meaning and affective meaning. The visual elements in Dunkin Donuts advertisement has affective meaning, in this case those visual elements are intended to be able to affect the readers or consumer to try the Dunkin Donut's product

\section{References}

[1] G. Dyer, Advertising as Communication. London: Routledge, 1993.

[2] G. Leech, Semantics: The Study of Meaning, Second. Harmondsworth: Penguin Book Ltd, 1985.

[3] G. Brown, G and Yule, Discourse Analysis. London: Cambridge University Press, 1983.

[4] P. Manning, Semiotics of Drink and Drinking. USA: Bloomsbury Academic, 2012.

[5] T. A. Sebeok, Signs: An introduction to semiotics, 2 Ed., vol. 26, no. 4. Toronto: Univeristy Of Toronto Press, 2001.

[6] N. Mzoughi and S. Abdelhak, "The impact of visual and verbal rhetoric in advertising on mental imagery and recall," International Journal of Business and Social Science, vol. 2, no. 9, pp. 257-267, 2011. 
[7] E. Negm and P. Tantawi, "Investigating the Impact of Visual Design on Consumers' Perceptions towards Advertising," International Journal of Scientific and Research Publications, vol. 5, no. 1, pp. 2250-3153, 2015.

[8] S. Mzoughi, N., Abdelhak, "Visual and verbal rhetoric in advertising," International Journal of Business and Management Studies, vol. 1, no. 4, pp. 413-422, 2012. 